\title{
Radio telescopes
}

\section{F.Tercero}

Observatorio de Yebes (Observatorio Astronomico Nacional)

Apdo. 148.19080 Guadalajara

SPAIN

E-mail: f.tercero@oan.es

Basic concepts in Radio Telescopes are presented to join the designer's view of the antenna engineer with the radio-telescope user. Single dish radio-telescopes based in the paraboloid and cassegrain systems are compared.

2nd MCCT-SKADS Training School Radio Astronomy: fundamentals and the new instruments Sigüenza (Spain)

August $26^{\text {th }}-$ September $4^{\text {th }} 2008$ 


\section{Introduction}

The invention 400 years ago of the optical telescope changed the known universe giving a new view of the sky. The detector used for the observation of the sky was the human eye, however the interposition of the telescope between the naked eye and the universe gave a magnified view of astronomical objects that, although were still distant, could give details never seen before and consequently innumerable stars and celestial objects were discovered. Optical astronomy was developed in conjunction with optical technology but little attention was invested in exploring the electromagnetic spectrum widely. The development of the TransAtlantic Radio communications led to the belief that interferences in the communication signal, which were registered at the same hours every day, could not have originated from the earth. Finally radio emission from our galaxy was identified. Again the telescope (in this case an antenna that formally was the first radio telescope) was the fundamental key to reveal a new cold and hidden universe.

The telescope can be understood as an instrument that collects and concentrates energy from the observed source and gives high angular discrimination. Both objectives can be fulfilled by constructing telescopes whose size is as large as is practically possible in terms of wavelength. Moreover it is an electromagnetic antenna, a simple definition of which is that is a transducer between the free space propagation and the guided propagation for the electromagnetic waves. The radio spectrum window is much bigger than the optical one, from the $\mathrm{Hz}$ waves to the millimeter waves up to $300 \mathrm{GHz}$. Many antenna designs exist in the broad radio band, where each specific design fulfils the requirements of the frequency band of observation. Due to the very large bandwidth of the RF spectrum it is impossible to have broadband antenna design that works optimally in all the radio spectrum, consequently the aspect and operation principle of antennas changes from dipoles to big aperture antennas at low and high frequencies respectively.

A general description of the parameters that determine the electrical behavior of an antenna will be shown. Aperture antennas, reflector and horn antennas, will also be explained in more detail with the aim of giving understandable concepts (avoiding a rigid theoretical analysis) that can be transferred easily from the antenna engineer's viewpoint to the radio telescope user. These reflector antennas are widely used from the $\mathrm{GHz}$ up to the Far Infrared and represent a nice example of where the optical and microwave technology merge. 


\section{Fundamentals of the detection EM waves in the radio domain}

The sky brightness is an adequate measure of the intensity of resolved source. It is defined, in a specified direction as the flux density per unit solid angle of the source, $\mathrm{W} \mathrm{m}^{-2} \mathrm{~Hz}^{-1} \operatorname{strad}^{-1}$. The spectral distribution of flux density differs for different radio sources. The sources radiate by different emission mechanism and have been divided into two main groups, thermal and nonthermal emitters.

The spectrum of thermal radiation from a black-body is given with enough accuracy at the frequencies and intensities normally encountered in radio astronomy by Rayleigh's classical approximation, derived from the Planck's Law.

$B_{v}(T)=\frac{2 h v^{3}}{c^{2}} \frac{1}{\exp \left(\frac{h v}{k T}\right)-1} \quad$ Planck's Law

Eq. 1

$B_{v}(T)=\frac{2 k T v^{2}}{c^{2}}=\frac{2 k T}{\lambda^{2}} \quad$ Rayleigh-Jeans approximation when $(h v \ll k T) \quad$ Eq. 2

In the case of sources that radiate like black bodies the frequency is squared, (e.g. Moon and Planets without atmosphere). In other cases this exponent changes, for example, in the case of transparent gas clouds the spectrum is flat due to the dependence of the frequency of the optical depth. In any case, from the antenna engineer's viewpoint, the use of this brighness spectrum lets us define an antenna temperature, that is the case of sources that radiate as black bodies, that it is the object temperature.

\subsection{Antenna Basics}

Almost any type of antenna can described with a few concepts that can be defined (or measured or calculated) independently of the design of the antenna. They can be applied both for dipoles and aperture antennas. The most important concepts relative to the radio telescopes are mentioned below.

Radiation Pattern: is the mathematical function that represents the radiation (or reception) properties of the antenna in the space. It is a 2 dimensional function (usually in spherical thetaphi coordinates) and it is independent of the distance to the antenna. In practical cases diagrams with the phi coordinate fixed are shown (at 0 and 90 degrees). It usually has several lobes that are the directions where the radiation is received strongly. The antenna is usually pointed with the main lobe at the source, and the radiation received with the secondary side lobes is undesired because it is not pointing to the source under observation. The measurement of the width of the beam between the $-3 \mathrm{~dB}$ points is usually known like HPBW (Half Power Beam Width) 


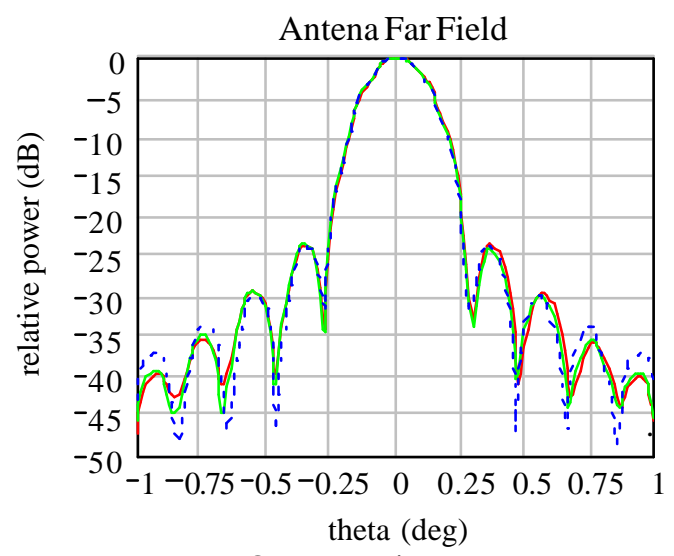

Fig 1 Example of radiation pattern normalized at OdB

Directivity: is a measurement of the radiation of the antenna at maximum intensity in comparison with an isotropic radiator (radiates equally in all directions). This figure is not much used in astronomy. However the concept is widely used because the more directive is an antenna the narrower is the main lobe of the radiation pattern.

Polarization: Is the polarization of the wave received by the antenna. Linear and elliptical polarization are the bases for describe any other polarization. In linear polarization the horizontal and vertical are the orthogonal bases components, while in the elliptical polarization are the right and left polarizations. The antennas can be polarization sensitive or not. In any case, if we want to detect both polarizations the two polarizations are separated in two separate receiver chains.

Effective Area: Is the ratio of the available power at the terminal of a receiving antenna to the power flux density of a wave incident to the antenna. It is independent of the geometrical area and can be defined for any type of antenna

Antenna Efficiency: Is the ratio of the effective area to the physical. Intuitively, it has physical sense for the aperture antennas where a real physical aperture can be defined. It is always less than 1 and it takes into account all the inefficiencies that can be found in an antenna (e.g. ohmic losses, impedance mismatches, cross-polarization)

$$
\eta=\frac{A_{e f f}}{A_{p h y}}
$$

Antenna Temperature: The antenna receives power from its environment and this power is delivered to the antenna terminals. If an equivalent resistor in thermal equilibrium at temperature $\mathrm{T}$ is used, the equivalent temperature of the resistor is directly related to the surface temperature of the observed source if the Rayleigh-Jeans approximation is used. 


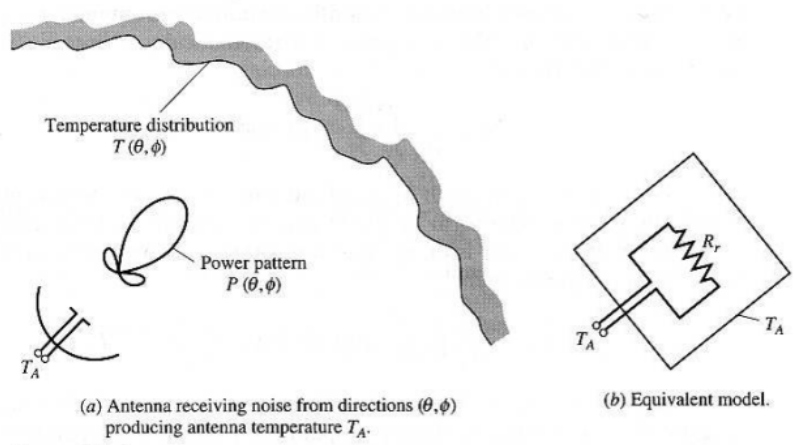

Fig 2 Equivalent model of antenna

The temperature associated with the power detected depends on the relation between the relative solid angles of antenna and source. If the source size is smaller than the antenna beam solid angle (and its temperature is constant), the antenna temperature is the source temperature weighted by the solid angles ratio. If the source is more extensive than the antenna solid angle the antenna temperature is directly the source surface temperature.

$$
\begin{aligned}
& T_{A}=\frac{1}{\Omega_{A}} \int_{4 \pi} T_{\text {source }}(\theta, \phi) P(\theta, \phi) d \Omega \\
& \Omega_{\text {source }}<<\Omega_{A} \quad T_{A}=\frac{\Omega_{\text {source }}}{\Omega_{A}} T_{\text {source }} \\
& \Omega_{\text {source }}>>\Omega_{A} \quad T_{A}=T_{\text {source }}
\end{aligned}
$$

Bandwidth: The antenna fulfils the requirements in a limited bandwidth. All the described parameters are frequency dependent.

\section{Single Dish Radio Telescopes}

The practical implementation of an antenna (or radio telescope) depends mainly of the frequency range. For the lower frequencies the wire antennas are the most common, while for the higher frequencies it is more usual to find reflector type antennas. These kinds of antennas concentrate the radiation in one single point where an additional antenna is used as a feeder for the system. The cutting edge between low and high frequencies is not sharp with the end result that is usually necessary to mix technologies from different frequency regimes. It is quite typical to find continuous surface reflectors fed by wire antennas. 

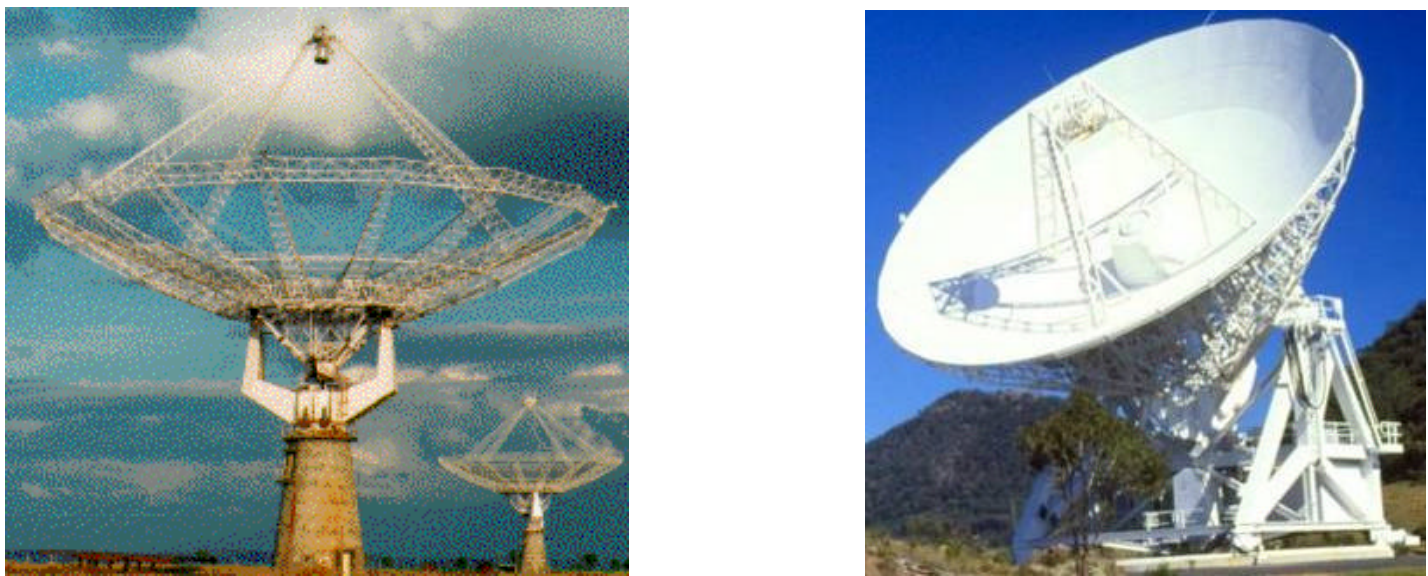

Fig 3 Example of continuous and wired reflector antenna

\subsection{Paraboloid Reflector}

The mathematical surface of Paraboloid Reflector is a parabola of revolution with focal length $\mathrm{F}$ and diameter $\mathrm{D}$. It has two important geometrical properties:

All rays leaving the focal point $(F)$ are collimated after reflection parallel to the revolution axis All path lengths from $F$ to the reflector and on the aperture are equal $(2 F)$ for any ray

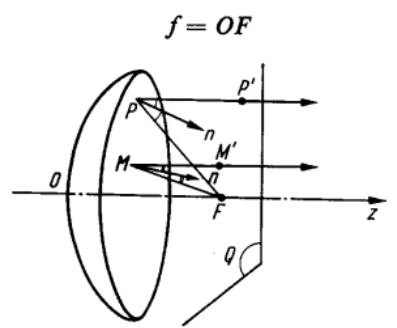

Fig 4 Paraboloid reflector geometry

The paraboloid reflector works as a phase-transformer by transforming spherical waves that come from the focal point to flat phase-front waves after reflecting the parabola (commonly known as the prime focus). Consequently a field distribution is presented in the physical aperture of the reflector where the amplitude distribution is determined by the radiation pattern of the feed that it is placed in the focal point $(F)$. The radiation pattern of the reflector antenna is related to the aperture field distribution. As the diameter of the aperture is usually a large number of wavelengths, the beam-width (half power beam-width) of this antenna is very narrow and can be calculated approximately with $\lambda / \mathrm{D}$ (wavelength and parabola diameter)

The amplitude distribution in the reflector aperture depends on the amplitude distribution of the feed used in the focal point. The ideal feed should illuminate the reflector surface with a constant amplitude distribution and this ideal feed should radiate nothing outside of the stereoangle subtended by the reflector. The aperture efficiency for this ideal feed is 1 , that means that 
all the power intercepted by the aperture is efficiently transferred to the receiver without any loss.

Real feeds are far from having this behaviour, and they present a radiation pattern similar to a Gaussian distribution. This amplitude distribution is not constant on the reflector surface and is not null outside of the reflector. These two effects can be evaluated to analyze the impact in the antenna efficiency. The amplitude distribution of the feed is characterize with the feed illumination taper, that is the amplitude level of the feed radiation pattern in the edge of the reflector respect the maximum, that it is in the axis of revolution. If the radiation pattern is normalized to 1 at the maximum, the taper is lower than 1 (or negative if it is in $\mathrm{dB}$ ). The concept of taper is shown in the figure.

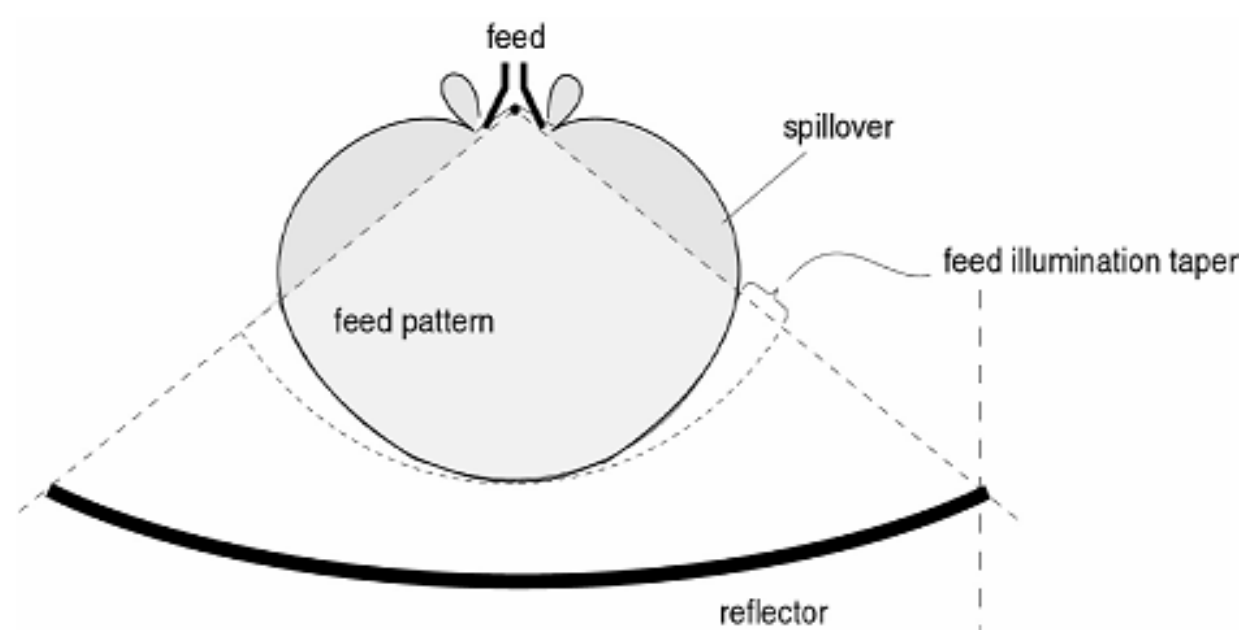

Fig. 5: Taper and Spillover concepts

The aperture efficiency depends strongly of the taper (even more that the amplitude distribution profile). If the taper is small (small difference with maximum), the amplitude distribution along the reflector is similar to the uniform distribution and the taper efficiency (defined as the ratio of the effective area vs. physical area because the non-uniformity amplitude distribution) is close to 1 , however there is much power of the feed that it is not reflected by the reflector and it is useless. This effect is known as spillover and the spillover efficiency (defined as the ratio of the effective area vs. physical area because the spillover power) drops to 0 . A part from the loss of efficiency, the spillover is looking directly to the ground at $300 \mathrm{~K}$, increasing the antenna temperature with an undesired signal contributing to the noise of the receiver. On the other hand, if the taper is big (big difference with maximum), we have the opposite effect, little power is reflected outside the antenna (spillover efficiency close to 1) but the amplitude distribution along the reflector is not uniform and taper efficiency is close to 0 .

The optimum figure of the taper is between -10 and $-12 \mathrm{~dB}$ depending of the amplitude distribution. In this case we have 0.9 of efficiency for both spillover and taper efficiency. The product of both is named illumination efficiency, and its optimum value is 0.81 . This figure is difficult to increase with standard feeds and reflectors and it represents a theoretical limit set 
only by the feed illumination that in reality decrease due to asymmetries, cross-polarization, losses, mismatches, positioning errors, manufactured reflector surface precision...
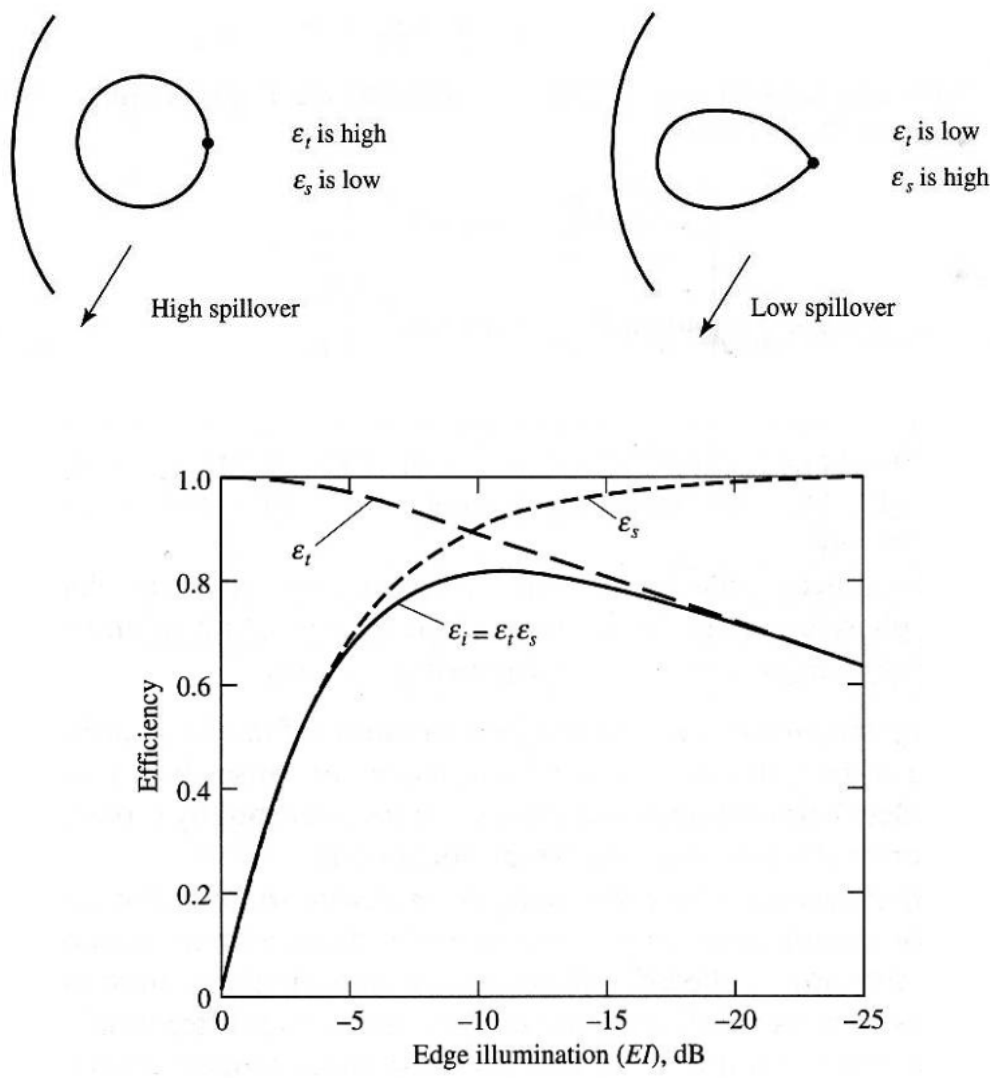

Fig 6 Taper and spillover efficiencies that maximizes the illumination efficiency

Once the feed has beeen chosen to have between -10 and $-12 \mathrm{~dB}$ taper at the edge of the reflector, the design parameter of a paraboloid reflector is the focal length and the diameter. The diameter is usually fixed by the cost of construction and the angular resolution required. The focal distance is usually analyzed with the diameter of the reflector, being the important figure the f-number (ratio between focal and diameter). The f-number determines the aspect ratio of the antenna and usual figures are found between 0.3 and 1. 


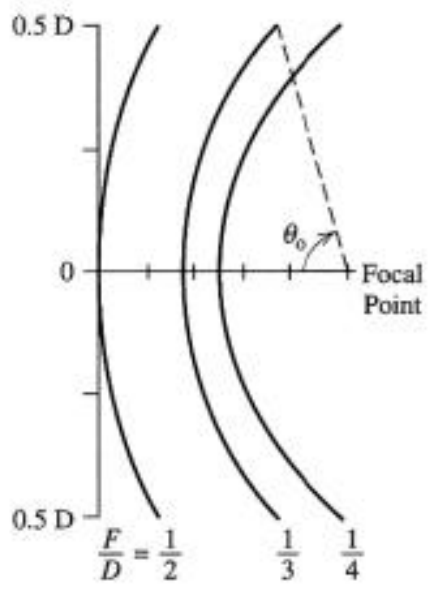

(a) Shapes for common $F / D$ values.

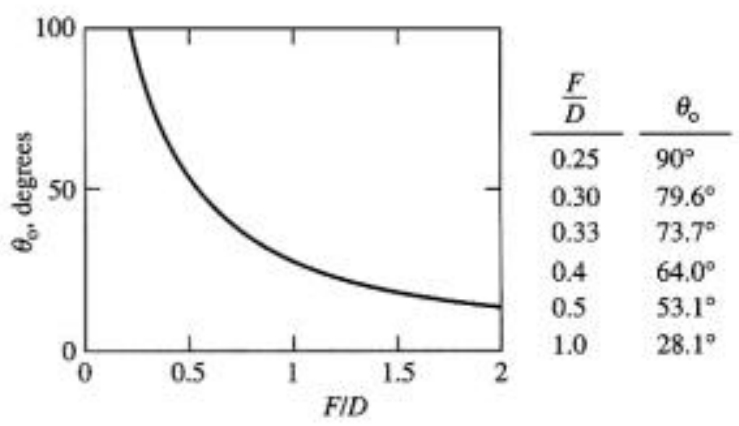

(b) Angle from the reflector axis to rim $\theta_{0}$ versus $F / D$

Fig 7: Aspect ratio of paraboloid reflector depending off-number. Illumination subtended angle in paraboloid reflector.

The f-number determines some important electrical properties of the antenna that can be summarized as follows:

Low $f$-number. The shape is curved and the feed is near of the reflector vertex. The feed used must be of low directivity. Cross-polar is generated in the reflector and the system is more sensitive to the axial and lateral displacements of the feed respect the focal point.

High f-number. The shape is more flat and the feed is far from the vertex. Cross-polar is lower and the system has a higher immunity to misalignments.

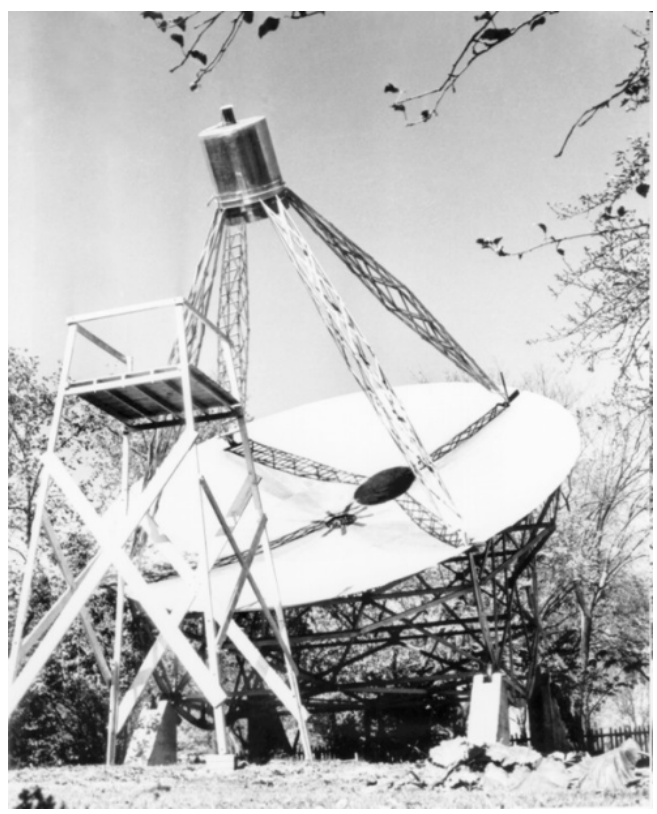

Fig 8: Example of large F/D antenna 
The prime focus paraboloid reflector has the following important limitations improved by the dual reflector systems:

1. The feed and receiver are not easily accessible and long cables must be used to transport the signal.

2. f-number range limited by the construction of tetrapod

3. Feed spillover sees the ground temperature $300 \mathrm{~K}$

4. The low f-number increases the cross-polar radiation pattern and it makes the system very sensitive to the feed positioning errors. The Fig.9 shows how the efficiency reduces quickly with axial and lateral errors in feed positioning

FAD

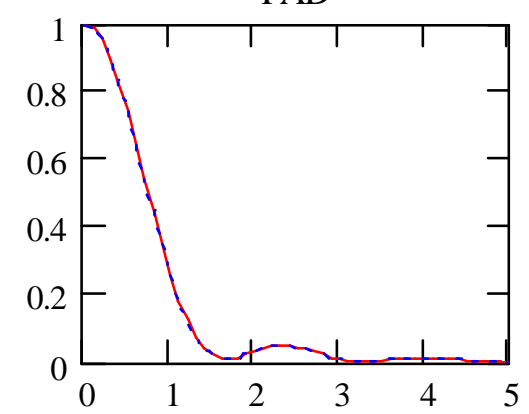

FLD

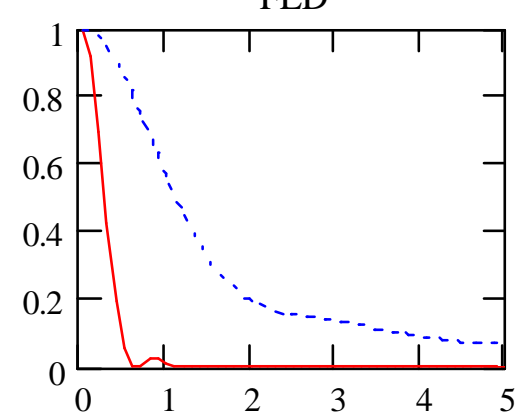

Fig 9: Aperture Efficiency reduction vs. Feed Axial Displacements (FAD) and Feed Lateral Displacements $(F L D)$ in wavelengths for a $F / D=0.375$ paraboloid reflector. At high frequencies, for instance $100 \mathrm{GHz}$, an error of the feed positioning of $3 \mathrm{~mm}$ means that aperture efficiency drops to $20 \%$ (axial error) or 50\% (lateral error. Dotted line: antenna pointed to the new maximum).

\subsection{Dual Reflector. Cassegrain Antenna}

The Cassegrain reflector is the combination of paraboloid and hyperbolic reflectors where the axis of revolution of both surfaces is coincident. The paraboloid reflector has focal distance $\mathrm{F}$ while the hyperbolic reflector has one of its foci coincident with the paraboloid reflector focus. With this configuration any ray parallel to the axis of the antenna is reflected by the paraboloid dish towards the F point, however the hyperbolic reflector has the property of reflect the rays that point to $F$ towards F'. The feed is placed at the F' point, this position is far from the parabola focus $F$. 


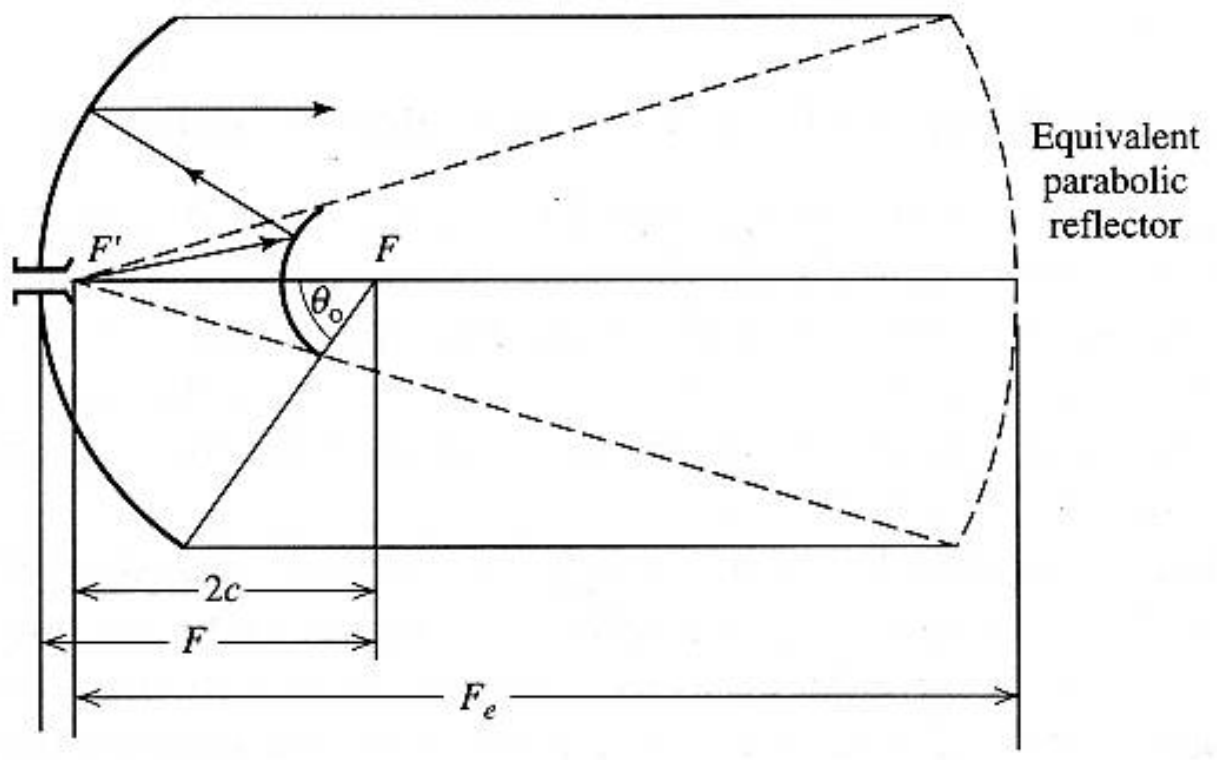

Fig 8: Cassegrain geometry and Equivalent Paraboloid Reflector.

The properties of the Cassegrain antenna can be derived from an equivalent paraboloid reflector with the same diameter of the main reflector and new equivalent focal length that is the focal length of the primary magnified by the secondary. The magnification factor depends on the eccentricity of the hyperbolic reflector e.

$$
\begin{aligned}
m & =\frac{e+1}{e-1} \\
F_{e} & =m F
\end{aligned}
$$

As an example we present the 40m Radio-telescope operated by the National Spanish Observatory in Spain (www.oan.es). 


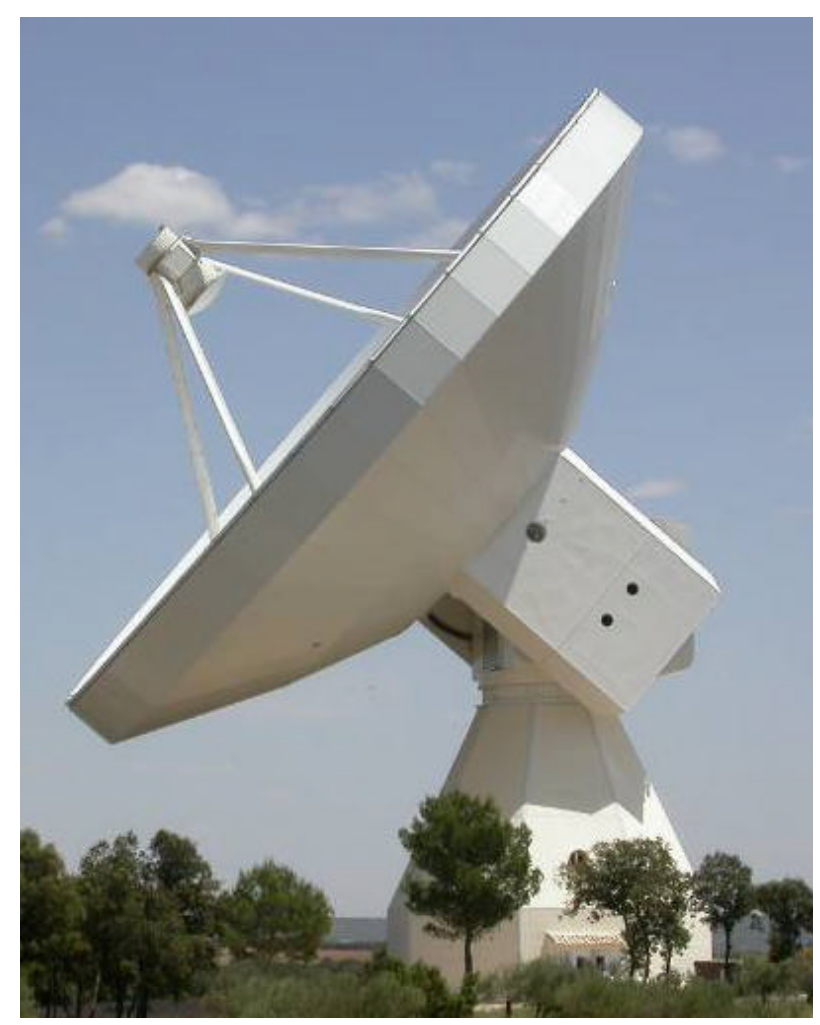

\begin{tabular}{|l|l|l|r|}
\hline Dp & diameter primary & {$[\mathrm{m}]$} & 40.000 \\
\hline $\mathrm{Fp}$ & focal primary & {$[\mathrm{m}]$} & 15.000 \\
\hline Fp/Dp & f-number primary & & 0.375 \\
\hline $\mathrm{Ds}$ & diameter secondary & {$[\mathrm{m}]$} & 3.200 \\
\hline $\mathrm{e}$ & eccentricity & & 1.099 \\
\hline $\mathrm{m}$ & magnification & & 21.090 \\
\hline $\mathrm{Fe}$ & focal equivalent & {$[\mathrm{m}]$} & 316.350 \\
\hline $\mathrm{Fe} / \mathrm{Dp}$ & f-number equivalent & & 7.909 \\
\hline
\end{tabular}

Fig 9: The 40m radiotelescope of Yebes and its main optical parameters

It illustrates how the addition of the secondary helps with all the limitations associated with the low f-number of the paraboloid reflector. The f-number of the primary is always limited by the construction of tetrapod but it is not the relevant electrical parameter in Cassegrain configuration because it is factored by the magnification.

The cassegrain antenna improves with respect the prime focus paraboloid reflector by

1. The feed and receiver are easily accessible. They can be even placed under the parabola surface in an real receiver's cabin

2. f-number(equivalent) is not limited by construcution of the tetrapod.

3. Feed spillover sees the sky temperature $3 \mathrm{~K}$ 
4. The high f-number(equivalent) makes the system insensitive to the feed positioning errors. The Fig.10 shows how the efficiency keeps constant with small positioning errors.
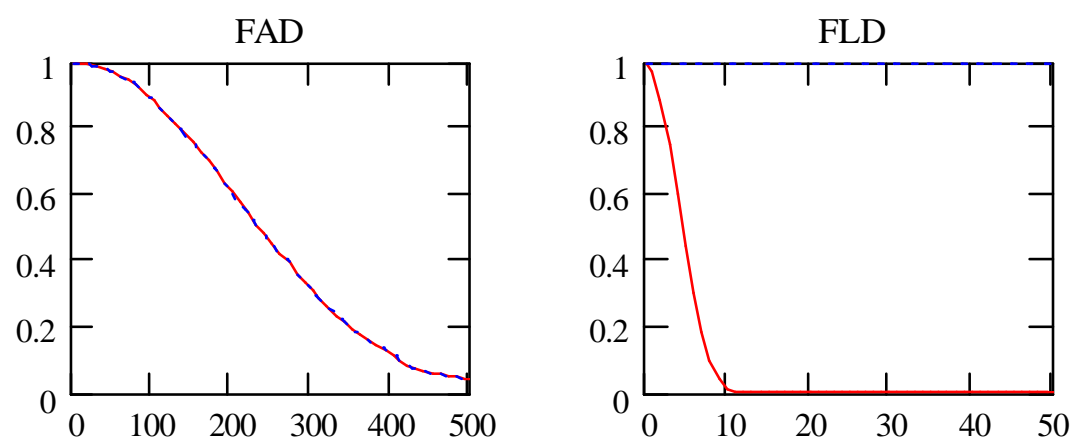

Fig 10. Aperture Efficiency reduction vs. Feed Axial Displacements (FAD) and Feed Lateral Displacements (FLD) in wavelengths for a Fe/D=7.909 cassegrain reflector. Several wavelengths of axial and lateral error for the feed positioning are allowed without loss of efficiency

\subsection{Effective Area and Ape rture Efficiency.}

As pointed in section 3.1 the most important contribution for the calculation of the effective area of a single dish reflector is the taper and spillover (illumination) contribution of the feed with the reflector. Many other factors reduce the effective area (and consequently the antenna efficiency):

Phase Errors. The wavefront is not always spherical (the wave seems to come from a single point called the phase centre). Usually it is disturbed in the two main planes (phi equal to 0 degrees and 90 degrees) defining different phase centre points for both planes. The Phase centre definition is also not well defined across the entire bandwidth definition of the feed. Additionally mechanical misalignments can result in large distribution phase errors in the reflector. The effect depends on the feed design and the magnitude and type of the misalignments. However in practice in dual reflector systems it can be reduced drastically to negligible figures. In Eq. 7 is denoted by $\eta_{p}$.

Cross Polar. The feed do not only radiates one pure polarization. The power that is lost from one polarization to the other (the effect is the same in linear and circular polarization) is seen like a lost of antenna effective area. The reflector also transform form one polarization to the other due to the curvature of reflectors mainly. Eq. 7 is denoted by $\eta_{x}$.

Blockage. In axially rotated systems (both for single and dual reflectors) the feed (or the sub-reflector in the case of the dual reflector systems) blocks the aperture reducing the incident power in the reflector. This effect can reduce the aperture efficiency of the antenna up to 5\%. In Eq. 7 is denoted by $\eta_{b}$. 
Surface errors. The construction of big dishes is not a trivial problem. The implementation of the mathematical paraboloid and hyperbolic surface cannot be done with perfect accuracy. The dishes large size is responsible for distributed surface errors, giving aberrations like astigmatism (different focus for different phi-planes). This kind of errors must be considered in the mechanical design of the antenna, adopting the homology design principle, which ensures that the paraboloid shape is always guaranteed but with different primary focus, depending of the elevation. The focus is actively aligned by the re-positioning of the sub-reflector.

The surface quality of the panel manufacturing also affects the performance giving the high frequency limitation of the antenna. The aperture efficiency due to the random errors in the surface is given by the Ruze's rule. $\varepsilon$ is the r.m.s. of the surface of the reflector, it can be easily calculated that an error of $\lambda / 16$ reduces the antenna efficiency to $50 \%$. For instance a $100 \mathrm{GHz}$ antenna needs a 180 microns surface error over the entire antenna surface to have a maximum antenna efficiency of $40 \%$ of its physical area (taking into account only the illumination and surface efficiency) . In Eq. 7 is denoted by $\eta_{s}$.

$$
\eta_{s} \approx e^{-\left(\frac{4 \pi}{\lambda}\right)^{2}}
$$

Finally all the antenna efficiencies must be multiplied to calculate the total antenna efficiency.

$$
\eta_{a p}=\eta_{t} \eta_{s p} \eta_{p} \eta_{x} \eta_{b} \eta_{s}
$$

\section{Conclusions}

Basic antenna definitions are presented in relation to paraboloid and Cassegrain antennas that are widely used as radio-telescopes. The close dependence of the feed radiation pattern and the antenna efficiency is presented in terms of taper and spillover concepts for paraboloid reflectors. The relevance of the focal length in the reflector systems is highlighted in relation to the limited focal length of prime focus paraboloid systems. The increase of the focal length and the improvement of the equivalent noise temperature of the receiver is demonstrated as the motivation for the development of dual-reflector radio-telescopes. The Cassegrain antenna is demonstrated to improve the performance of antenna efficiency over that of the prime focus paraboloid antenna with a little extra cost. Finally, the aperture efficiency is presented as a sum of all the efficiency characteristics of the radio-telescope.

\section{References}

[1] “Radio Astronomy. $2^{\text {nd }}$ Edition”. J.D. Kraus. Cygnus-Quasar Books.1986

[2] "Methods of Experimental Physics vol.12-B. Radio Telescopes". M.L. Meeks (Ed.). Academic Press. 1976 


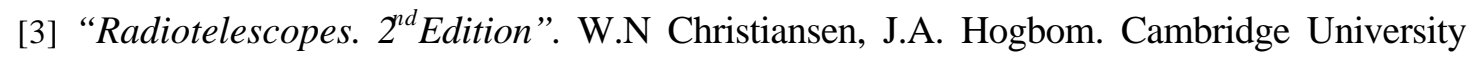
Press. 1985

[4] “Antenna Theory and Design”. W.L. Stutzman, G.A. Thiele. John Wiley Inc. 1998

[5] "Microwave Horns and Feeds". A.D. Olver, P.J.B. Clarricoats, A.A. Kishk, L. Shafai. IEE Press. Electromagnetic Waves Series 39. 1994

[6] “Reflector Antennas”. A.W. Love (Ed.). IEEE Press. 1978

[7] “Quasioptical Systems”. P.F. Goldsmith. IEEE Press. 1998

[8] "Interferometry and Synthesis in Radio Astronomy". A.R. Thompson, J.M. Moran, G.W. Swenson. Krieger Publishing Company. 1994 